\title{
Pain in People with Advanced Dementia: The Opinions of Kazakh Medical Students
}

This article was published in the following Dove Press journal:

Journal of Pain Research

\author{
Slawomir Tobis (iD) ${ }^{\prime}$ \\ Agnieszka Neumann- \\ Podczaska (iD ${ }^{2}$ \\ Lyudmila Yermukhanova (iD ${ }^{3}$ \\ Gulnara Sultanova (1D ${ }^{4}$ \\ Gulnara Kurmanalina $\mathbb{D}^{5}$ \\ Kerbez Kimatova (iD ${ }^{3}$ \\ Marzena Dworacka $\mathbb{D}^{6}$ \\ Katarzyna Wieczorowska- \\ Tobis (I) $^{2}$ \\ 'Department of Occupational Therapy, \\ Poznan University of Medical Sciences, \\ Poznan, Poland; ${ }^{2}$ Geriatric Unit, \\ Department and Chair of Palliative \\ Medicine, Poznan University of Medical \\ Sciences, Poznan, Poland; ${ }^{3}$ Department of \\ Public Health and Health Care, West \\ Kazakhstan Marat Ospanov State Medical \\ University, Aktobe, Kazakhstan; ${ }^{4}$ West \\ Kazakhstan Marat Ospanov State Medical \\ University, Aktobe, Kazakhstan; \\ ${ }^{5}$ Department of Internal Medicine, West \\ Kazakhstan Marat Ospanov State Medical \\ University, Aktobe, Kazakhstan; \\ ${ }^{6}$ Department of Pharmacology, Poznan \\ University of Medical Sciences, Poznan, \\ Poland
}

Correspondence: Slawomir Tobis

Department of Occupational Therapy, Poznan University of Medical Sciences, ul. Swiecickiego 6, 60-78I Poznan, Poland Tel +486I 8546573

Email stobis@ump.edu.pl
Purpose: The medical students' attitude toward pain in people with advanced dementia, while constituting an important factor in care, has rarely been assessed to date. The aim of our study was thus to perform such assessment in medical students in Kazakhstan, to enable an improvement of the existing curriculum (like we previously did in Poland).

Materials and Methods: We analyzed the knowledge about pain using a short anonymous questionnaire, which was completed by 112 students of the Medical University of Aktobe, Kazakhstan.

Results: On average, students listed symptoms of $1.4 \pm 1.2$ (out of 6 analyzed) pain areas (median 2.0). The symptoms related to changes in mental status were suggested the most often (57 students: 50.9\%). The students who indicated these symptoms also listed a higher number of symptoms from the remaining domains $(1,1 \pm 1.0$ [median 1.0] vs $0.6 \pm 0.8$ [median 0.0]; $\mathrm{p}<0.01)$. Observational methods in the assessment of the severity of pain in people with dementia were indicated by 44 students $(39.3 \%)$, but only one participant $(0.9 \%)$ was able to name an observational scale for pain assessment. Correct answers regarding pain treatment rules were presented by 18 students $(16.0 \%)$, and the answers of the next 47 participants $(42.0 \%)$ were very general but suggested the same treatment no matter what the cognitive status.

Conclusion: The study revealed gaps in the knowledge of Kazakh medical students regarding pain in advanced stages of dementia. Demographic changes, combined with the coexistence of pain with dementia, indicate that medical students worldwide must have sufficient knowledge and skills to adequately care for the continually growing number of people with these conditions. It is imperative in countries like Kazakhstan, where the dementia burden was unrecognized until now, but it will blow up in the near future.

Keywords: education, pain, advanced dementia, medical students, knowledge

\section{Introduction}

Population aging poses numerous concerns for health care systems as the need for medical care naturally increases with age. Among others, care for subjects with dementia presents particular challenges. The global number of people living with dementia more than doubled during the last 40 years due to increases in the aging of societies. ${ }^{1}$ Satisfying the needs of this group requires knowledge of the specificity of their problems. One of the most underdiagnosed (consequently, also undertreated) symptoms in subjects with dementia is pain.

Appropriate and accurate pain assessment is essential for adequate and effective pain treatment. Literature reports show deficiencies in pain detection, which result in lack or (at least) inappropriateness of pain treatment. According to data available 
from all over the world, the issue persists with older persons in general, but especially with those with dementia. They receive fewer or lower doses of analgesics than their counterparts without cognitive impairment, even in the same painful situations. ${ }^{2-5}$

As severe dementia is commonly associated with loss of communication ability, pain assessment is particularly challenging. Individuals with this condition are not able to give reliable self-reports or to provide retrospective data about the onset, duration, and intensity of their pain. Furthermore, because subjects with severe dementia do not comprehend questions related to the estimation of their pain, observation, and detection of pain-related behavior are the most suitable approach to the identification of pain. The care staff must be capable of performing such assessments, without which no adequate pain therapy is possible. Moreover, it is imperative to differentiate pain behavior from other need driven ones. ${ }^{6}$ Therefore, proper education of future staff is a crucial issue. As reported by Zwakhalen et al, medical students had little knowledge of the use of analgesics and their dosage. ${ }^{7}$ Barber and Gibson observed an uncertainty of physicians regarding the optimum management of pain in dementia, ${ }^{8}$ which was related to their beliefs about age-related changes in drug metabolism. Notably, knowledge gaps in the pharmacological treatment of pain may result in its undertreatment.

In Kazakhstan, the subpopulation of those who are 60 years and older presents $11.1 \%$ of the entire population, whereas, eg in Germany $-28.0 \%$ and in Japan $-33.4 \%$ (2017). ${ }^{9}$ Then again, the birth rate in Kazakhstan is 2.6 children/female, whereas in Taiwan or Portugal - only 1.2. ${ }^{10}$ Thus, compared to many other countries all over the world, Kazakhstan's society is still relatively young. However, a demographic prognosis indicates that the population of Kazakhstan will also enter the trajectory of aging. $^{11}$

Previously, we demonstrated that the knowledge of medical students on pain treatment in subjects with dementia, in a country with a high prevalence of the condition and rapidly aging society (Poland), deserved an improvement. $^{12}$ Based on a similar methodology, we decided to perform an analogous study in a younger country, with a higher birth rate but also facing an acceleration of aging (Kazakhstan).

\section{Materials and Methods}

The study was approved by the Bioethical Committee of Poznan University of Medical Sciences in Poznan, Poland (the approval stated lack of features of a medical experiment). It is a continuation of the one performed among students of Poznan University of Medical Sciences, Poznan, Poland. ${ }^{12}$

Our short questionnaire about pain in advanced dementia was used with the 5th year medical students of Marat Ospanov Medical University in Aktobe, Kazakhstan, after their psychiatric course. There is no geriatric medicine course in the Kazakh curriculum; however, selected geriatric topics are covered during the course of internal medicine.

The students received a detailed explanation of the nature of the study. They were free to return an empty questionnaire at any time if they did not consent to participate. The completion of the questionnaire was deemed informed consent. All of the students asked agreed to take part in the survey. The study was anonymous. The participants were asked to fill the questionnaire without consulting any external resources, including the internet. It is thus justified to assume that the data obtained mirrored their knowledge.

The original questionnaire was created simultaneously in Polish and English; its details have already been presented. ${ }^{12}$ For the purpose of using it in Kazakhstan, it was translated into Russian (which is one of the two official languages in the country) by two independent persons with fluent knowledge of the Russian language. The translation was then verified and approved by two university teachers of Kazakh origin, to assure that the students in Kazakhstan understand the questionnaire correctly. All Kazakh students speak Russian, but as some of them had decided to be taught in Kazakh (they have a choice of the study language: Russian or Kazakh), we provided them with an option to select the language in which they provided their answers. One hundred twelve students participated in the study; 51 filled the questionnaire in the Kazakh language.

All answers were translated into English, as previously, by two persons who speak fluently both languages. The resulting translations were confronted and discussed until a consensus concerning the students' answers was reached. The qualitative data were analyzed by two independent researchers: a geriatrician and a clinical pharmacist, both of whom are graduates and members of the European Academy for Medicine of Aging (EAMA). Whenever the analysis yielded a discrepancy, the results were discussed to obtain consensus.

The questionnaire starts with a short introduction presenting a person with advanced dementia (including the indication that dementia in this patient is so severe that 
they might not understand the questions they are asked and thus not answer them correctly). The introduction is followed by three questions, all formulated in an open manner to avoid suggesting the answers.

The questions are:

1. What symptoms do you expect a patient with advanced dementia may have in case of pain?

2. How would you assess pain and its severity in a patient with advanced dementia?

3. Would you treat pain in a patient with advanced dementia in the same way as in a patient without dementia? If YES, please explain why? If NO, please explain why?

The aggregation of answers to the first question was based on the American Geriatric Society Panel on Persistent Pain in Older Persons, ${ }^{13}$ which states that the behavioral pain assessment chart should incorporate six domains:

- facial expressions (eg, slight frown, grimacing, any distorted expression),

- negative vocalization (eg, sighing, grunting, asking for help, verbally abusive),

- body language (eg, rigid body posture, fidgeting, increased pacing, restricted movement),

- changes in activity patterns (eg, refusing food, increase in rest periods, sudden cessation of common routines, increased wandering),

- changes in interpersonal interactions (eg, aggressive, resisting care, decreased social interactions, socially inappropriate, withdrawn), and

- mental status changes (eg, increased confusion, irritability or distress).

The answers to the second question were, after the initial analysis, aggregated according to the suggested types of pain scale: observational pain scales and pain scales recommended for use in the general population, including subjects with mild and moderate cognitive impairment (Visual Analog Scales - VAS), (Numerical Rating Scale - NRS), or (Face Pain Scale - FPS). ${ }^{14}$ The answers indicating the need to observe the patient and consult their family/caregivers to obtain information about their earlier state were additionally marked.

The answers to the third question were used to separate the respondents into three groups, regardless of the type of answer given (YES or NO), based on a detailed analysis of presented explanations:

- those who answered the question correctly, including those who gave a general, non-specific answer,

- those who answered the question incorrectly or partially correctly - ie, those who gave more than one explanation, and among them at least one was correct and at least one - incorrect (eg, NO - subjects with dementia should be treated differently from those without dementia because they have a different drug metabolism, but they are also not able to report side effects, so they must be carefully monitored for these effects),

- those who did not answer this question at all.

This approach builds upon our previous experience: sometimes students answering question 3 with NO (ie, that persons with advanced dementia should be treated differently than those without dementia), explained that this was due to, eg, the need to observe them for treatment effectiveness because they were not able to tell if the pain has disappeared spontaneously or after the administration of medication.

\section{Statistical Analysis}

The results were expressed as mean $\pm \mathrm{SD}$ and median +range due to a lack of normality of all analyzed parameters. To evaluate the normality of distribution, the Shapiro-Wilk test was applied. For selected parameters, the percentage of provided answers was calculated. A comparison between the groups was made with the Mann-Whitney test, and the frequency was compared with the Fisher exact test due to small groups. The statistical significance threshold was set at $\mathrm{p}<0.05$. In the case of a p-value between 0.05 and 0.10 , a trend with no statistical significance was observed.

\section{Results}

\section{Symptoms of Pain in People with Advanced Dementia in the Opinion of Medical Students}

The average age of 112 students who took part in the study was $22.2 \pm 1.3$ years (median 22 years; range: $20-29$ years). Among them, there were 36 men $(32.1 \%$ of all respondents). Only 15 students (13.2\%) declared having seen a person with advanced dementia. 
On average, the students listed symptoms from 1.4 \pm 1.2 out of 6 analyzed pain areas only (median 2.0, range $0-5$ ). No one listed symptoms from all six areas, and only two listed symptoms from five areas (Figure 1).

The most often suggested symptoms were those referring to mental status changes (57 students - 50.9\%). Symptoms from the remaining areas were indicated less frequently:

- facial expression: 14 students (12.5\%),

- body language: 23 (20.5\%),

- negative vocalization: 18 (16.1\%),

- changes in activity patterns: 38 (25.8\%),

- changes in interpersonal activity: 49 (33.3\%).

Notably, students who indicated mental status changes pointed to symptoms from a higher number of remaining domains than other participants $(1.1 \pm 1.0$ [median 1.0 , range $0-4$ ] vs $0.6 \pm 0.8$ [median 0.0 , range $0-3$ ]; $p<0.01$ ); in this, they more often mentioned symptoms in the areas of changes in activity patterns $(29.8 \%$ vs $9.1 \%$; $<<0.01)$ and negative vocalization $(24.6 \%$ vs $7.3 \%$; $<<0.05)$. The frequency of listing symptoms encompassing changes in interpersonal activity showed a nonsignificant trend (24.6\% vs $10.9 \%$; $\mathrm{p}=0.0836$ ).

Eight students indicated physiological changes as a clinical manifestation of pain (eg, fever, increased blood pressure, increased heart rate, fast breathing); two of them did not list any other symptoms.
Among the incorrect answers to the question about pain symptoms in people with advanced dementia, there was one from a student who suggested "the patient does not respond to pain," and another student expected patients to develop "deep neurological disorders."

\section{Assessment of the Severity of Pain in a Person with Advanced Dementia, in the Opinion of Medical Students}

Only one student $(0.9 \%)$ mentioned the name of an observational scale (PAINAID). However, as many as 44 students $(39.3 \%)$ indicated the necessity to observe the patient (ie, "Looking at facial expressions," "we assess pain based on the patient's facial expression and posture," "you need to determine pain by observing behavior; maybe he would cry, show a painful place or take a special position"). Seven students (6.3\%) suggested that family members should be asked (six of them also suggested to observe the subject).

Fourteen students (12.5\%) suggested that detailed physical examination (eg, palpation, auscultation) might be helpful when assessing the severity of pain in people with advanced dementia. Further 17 respondents (15.2\%) believed that the subject's face and movements should be observed during the examination ("during palpation in a painful place, facial expressions can be assessed; because of dementia, facial expressions must be assessed," "during the examination of the patient [palpation,

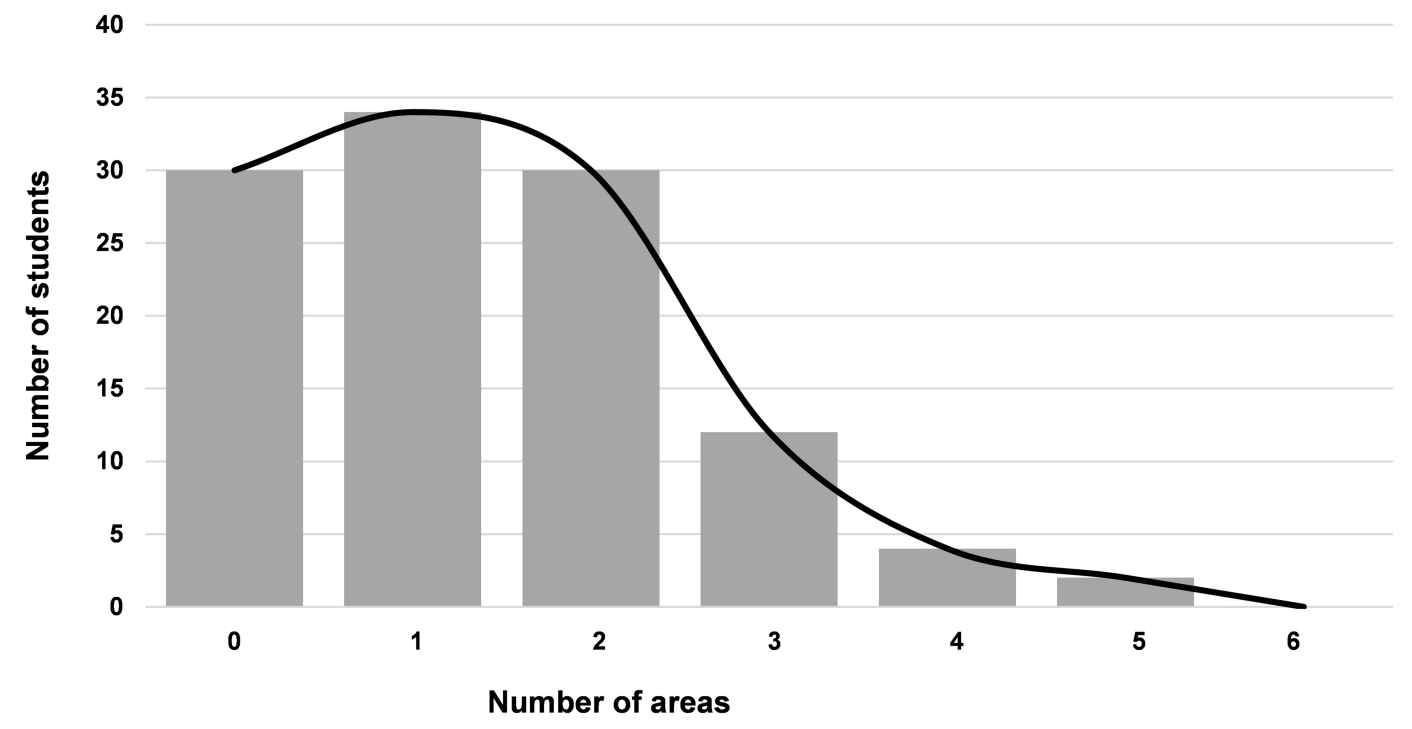

Figure I The number of studied students related to the number of areas from which they correctly indicated symptoms of pain. According to the American Geriatric Society Panel on Persistent Pain in Older Persons, behavioral pain assessment should incorporate six areas: facial expression, negative vocalization, body language, changes in activity patterns, changes in interpersonal interactions, and mental status changes. 
percussion, auscultation] we look at their reaction: cry, worry, scream"). Among them, there were six who also tended to do some laboratory tests, eg, to assess the inflammatory reaction.

On the other hand, 15 students (13.4\%) mentioned a point-rated scale (score 8-10 points out of 1-10 possible points), but nobody cited the name of the scale. Another five students $(4.5 \%)$ wanted to ask persons with advanced dementia additional questions about pain to assess if it is present and how its severity is.

\section{Treatment of Pain in People with Advanced Dementia, in the Opinion of Medical Students}

One in every six students $(18 ; 16.0 \%)$ explained pain treatment in persons with advanced dementia correctly, and $47(42.0 \%)$ gave a general answer (Table 1), which represents their positive attitude to the pain in people with advanced dementia.

Twenty-eight students (25.0\%) gave incorrect answers, and $19(17.0 \%)$ did not answer the question about pain treatment at all. The examples of pain treatment's characteristics are presented in Table 1.

\section{Discussion}

According to current prognoses, in countries of CentralEastern Asia, both life expectancy and the elderly subpopulation will be rapidly increasing. ${ }^{15}$ Aging and its consequences, therein the treatment of dementia patients, however, are issues that are still not sufficiently implemented. In the center of interest of health policy, also in Kazakhstan, there are other matters (eg, air pollution), which results in an extremely high prevalence of cardiovascular diseases with their mortality rate claimed the second-highest in the world. ${ }^{16}$ Our observation that only a dozen or so students, after completing their psychiatry course, declared having seen a person with severe dementia seems to be in line with this situation.

In many countries around the world, pain in people with dementia is underdiagnosed and undertreated. ${ }^{3,13,17,18}$ What is more, no research on the preparation of staff or students in this area is conducted; hence, no changes in education systems are introduced that would improve them. Following the forecasts regarding Kazakhstan's aging, we undertook a study to answer the question of how medical students are prepared to treat pain in people with advanced dementia.
Regarding pain detection in persons with advanced dementia, as many as $3 / 4$ students were able to list selected pain symptoms. Notably, the most frequently declared group of symptoms were those referring to deterioration in mental status - which seems to follow either the knowledge of prior behavioral patterns and permanent observation or seeking information from others (family members or further healthcare professionals). Therefore, mentioning these symptoms may indicate a group of students who have at least some orientation in the principles of diagnosing the patients in question in terms of pain. Thus, even though a majority of students did not see a person with advanced dementia, their level of knowledge about dementia alone was sufficient to navigate the area of pain symptoms in these persons properly. This is additionally confirmed by the fact that the group of students who indicated mental symptoms were also able to list a substantially higher number of symptoms, including those related to changes in activity patterns.

As for the methods of pain assessment in people with dementia, ${ }^{19}$ there were almost $40 \%$ of students who correctly pointed out the necessity of patient observation. Henceforth, even if only one student was able to correctly indicate an observation tool for assessing pain in persons with dementia, many had knowledge about the gist of observation when diagnosing these subjects. Notably, unidimensional pain scales (Visual Analogue Scale - VAS, Numerical rating Scale - NRS and Face Pain Scale - FPS) were relatively rarely indicated. Even if they should not be used in individuals with advanced dementia, they are considered valid as long as the person is able to understand them. ${ }^{20}$ Lack of official standards in the area of pain detection is pointed out in many countries. ${ }^{21}$ Moreover, according to Barry et al, even existing rules are underused or not used at all. ${ }^{22}$ It is thus essential to create general standards of conduct and familiarize a wide range of students and professionals with them, also in the countries where the topic of dementia has been widely discussed for years.

As far as the pharmacological treatment is concerned, we noticed a group of students who knew its rules. Some of them claimed that pain was the same regardless of the level of cognition. The others listed the Kazakh pain treatment protocol as a recommendation of the treatment scheme. This protocol contains comprehensive data on pain assessment and treatment, including pharmacotherapy in the general population, not addressing, though, the issue of pain in people with dementia. Taking into account the demographic prognoses for 
Table I Examples of Pain Treatment Characteristics Used by the Students to Explain if the Treatment of Pain in People with Advanced Dementia Should Be the Same or Different from Those Who are Cognitively Intact

\begin{tabular}{|c|c|c|}
\hline Correct Explanation & Wrong Explanation & $\begin{array}{l}\text { Too General an } \\
\text { Explanation }\end{array}$ \\
\hline $\begin{array}{l}\text { "Such patients require an individual approach } \\
\text { because they can't explain where and how } \\
\text { severe the pain is" }\end{array}$ & $\begin{array}{l}\text { "If the patient is in poor condition, or rather at the stage of } \\
\text { death, I see no point in continuing treatment. Of course, } \\
\text { I understand that this is inhumane, but from the medical point } \\
\text { of view, I consider it an unnecessary expense. At this stage, } \\
\text { I would advise relatives to spend their last days with the } \\
\text { patient" }\end{array}$ & $\begin{array}{l}\text { "It's my duty to treat every } \\
\text { patient" }\end{array}$ \\
\hline $\begin{array}{l}\text { "A patient with advanced dementia will forget } \\
\text { about medications, or may take them all at } \\
\text { once" }\end{array}$ & "Etiopathogenesis of pain is different" & $\begin{array}{l}\text { "We treat every patient } \\
\text { according to the protocol" }\end{array}$ \\
\hline $\begin{array}{l}\text { "The patient will not adhere to the } \\
\text { instructions; they may not take medications" }\end{array}$ & "Use of the treatment from narrow specialists" & $\begin{array}{l}\text { "Whoever the patient may be, } \\
\text { one should help him or at least } \\
\text { try" }\end{array}$ \\
\hline $\begin{array}{l}\text { "Such a patient cannot contact the outside } \\
\text { world" }\end{array}$ & "The patient feels no pain" & $\begin{array}{l}\text { "The doctor at first reflex } \\
\text { thinks how to help the ill and } \\
\text { relieve suffering" }\end{array}$ \\
\hline $\begin{array}{l}\text { "(Treat the same) because the patient feels } \\
\text { pain" }\end{array}$ & "If the patient is $80-90$ years old, no treatment is required" & $\begin{array}{l}\text { "The doctor's duty is to treat } \\
\text { the patient" }\end{array}$ \\
\hline $\begin{array}{l}\text { "Pain is the same as in a patient without } \\
\text { dementia; all you have to do is determine } \\
\text { exactly what hurts" }\end{array}$ & $\begin{array}{l}\text { "Pain and dementia often occur in older adults, so treatment is } \\
\text { not effective" }\end{array}$ & $\begin{array}{l}\text { "All patients are the same, and } \\
\text { it is our duty to treat every } \\
\text { patient" }\end{array}$ \\
\hline
\end{tabular}

Kazakhstan, there is an urgent need for a modification of the pain protocol to address pain management in dementia.

Among studied students, there were also those whose answers indicated both poor knowledge and improper attitude toward pain in dementia. Their answers bear traces of ageism and stigma around pain in dementia. One of them mentioned, "Pain and dementia often occur in older adults, so treatment is not effective." It must, however, be pointed out that stigma and misunderstandings around both aging and dementia are still present across the globe. According to the Dementia Attitudes Monitor, ${ }^{23}$ commissioned by Alzheimer Research in the UK, less than $1 / 4$ of British adults could adequately describe what exactly dementia means, and one in five incorrectly agreed with the statement "Dementia is an inevitable part of getting older." Additionally, more than $1 / 5$ of UK's adults agreed with the sentence "I would find it hard to talk to someone who has dementia." The issue of knowledge in the area of pharmacological pain management in people with dementia seems to be challenging for not only medical students but also physicians, eg, Barber and Gibson showed that physicians appeared to be uncertain about the optimum pain management in these patients. ${ }^{8}$

Our study also has some limitations. First, it is a singlecentered and cross-sectional analysis, hence extrapolating the data for the whole of Kazakhstan must be done with caution, even if the same pain protocol is implemented for the entire country. Also, the survey tool which was used to assess the students' knowledge about pain in people with severe dementia is not standardized. To the best of our knowledge, no such tool exists. Still, the questionnaire we used has been designed by the authors of this study themselves and contains open questions not to suggest answers. We successfully used this tool in our previous study to assess the Polish students' knowledge of pain in dementia; ${ }^{12}$ consequently, our curriculum has been changed based on its results.

The strength of our research is, however, that it is pioneering in defining the students' knowledge of pain and dementia. As far as we know, similar research was carried out only in Poland, and uncovering the education gap in Kazakhstan allows to adapt curricula to the needs in advance and prepare future professionals with a sufficient 
level of knowledge on pain management in people with dementia.

It should be emphasized that optimization of the geriatric curriculum is needed not only in Kazakhstan, where until now the burden of dementia was unrecognized but is likely to blow up very soon, as there is a worldwide lack of consensus among medical schools on how to teach about dementia. $^{24}$

\section{Conclusion}

The study revealed gaps in the knowledge of Kazakh medical students regarding pain in advanced stages of dementia. At the same time, in the wake of demographic changes, as well as prevalent coexistence of pain with dementia, medical students all over the world must have sufficient knowledge and skills to be able to adequately care for the continually growing number of people with that condition. It is imperative, not only in countries like Kazakhstan but also worldwide. The curricula of medical students should reflect these requirements - which means they need to be updated to stand contemporary challenges in the field of care for persons with pain and advanced dementia.

\section{Funding}

This research was supported by Poznan University of Medical Sciences (Funding number: 502-01-1114157006216); Ludmilya Yermukhanova, Gulnara Sultanova, Gulnara Kurmanalina and Kerbez Kimatova (WKMOMU) were supported by the Social Health Insurance Project, Republic of Kazakhstan (Contract No. SHIP-2.3/CS-02).

\section{Disclosure}

The authors report no conflicts of interest in this work.

\section{References}

1. Nichols E, Szoeke CEI, Vollset SE, et al. Global, regional, and national burden of Alzheimer's disease and other dementias, 1990-2016: a systematic analysis for the Global Burden of Disease Study 2016. Lancet Neurol. 2019;18(1):88-106. doi:10.1016/S1474-4422(18) 30403-4

2. de Souto Barreto P, Lapeyre-Mestre M, Vellas B, Rolland Y. Potential underuse of analgesics for recognized pain in nursing home residents with dementia: a cross-sectional study. Pain. 2013;154 (11):2427-2431. doi:10.1016/j.pain.2013.07.017

3. Nowak T, Neumann-Podczaska A, Tobis S, Wieczorowska-Tobis K. Characteristics of pharmacological pain treatment in older nursing home residents. J Pain Res. 2019;12:1083-1089. doi:10.2147/JPR. S192587
4. Nowak T, Neumann-Podczaska A, Deskur-Śmielecka E, Styszyński A, Wieczorowska-Tobis K. Pain as a challenge in nursing home residents with behavioral and psychological symptoms of dementia. Clin Interv Aging. 2018;13:1045. doi:10.2147/CIA. S157246

5. Holmerová I, Auer SR, Beránková A, Höfler M, Ratajczak P, Šteffl M. Cognitive status and use of analgesics and anxiolytics in residents of nursing homes in the Czech republic. Clin Interv Aging. 2018;13:2511. doi:10.2147/CIA.S188601

6. Kovach CR, Evans CR. Assessing Pain and Unmet Need in Patients with Advanced Dementia: The Role of the Serial Trial Intervention (STI). Handbook of pain and palliative care: Springer; 2018:127-143.

7. Zwakhalen SM, Hamers JP, Peijnenburg RH, Berger MP. Nursing staff knowledge and beliefs about pain in elderly nursing home residents with dementia. Pain Res Manag. 2007;12(3):177-184. doi:10.1155/2007/518484

8. Barber JB, Gibson SJ. Treatment of chronic non-malignant pain in the elderly: safety considerations. Drug Saf. 2009;32(6):457-474. doi:10.2165/00002018-200932060-00003

9. World Population Ageing 2017, Highlights. United Nations, New York, 2017. Available from: https://www.un.org/en/development/ desa/population/publications/pdf/ageing/WPA2017_Highlights.pdf. Accessed July 21, 2020.

10. World Population Review, Total Fertility Rate 2020. Available from: http://worldpopulationreview.com/countries/total-fertility-rate/. Accessed November 20, 2020.

11. Izekenova AK, Kumar AB, Abikulova AK, Izekenova AK. Trends in ageing of the population and the life expectancy after retirement: a comparative country-based analysis. J Res Med Sci. 2015;20 (3):250-252.

12. Neumann-Podczaska A, Tobis S, Yermukhanova L, WieczorowskaTobis K. Pain in advanced stages of dementia: the perspective of medical students. Medicina (Kaunas). 2019;55(5):116. doi:10.3390/ medicina55050116

13. Rospand M. AGS panel on persistent pain in older person. The management of persistent pain in older person. $J$ Am Geriatr Soc. 2002;50(6):205-224.

14. McClean WJ. Identifying and managing pain in people with dementia. Nurs Resident Care. 2003;5(9):428-430. doi:10.12968/ nrec.2003.5.9.11668

15. Global prevalence. Dementia Statistics Hub, Alzheimer's Research UK. Available from: https://www.dementiastatistics.org/statistics/glo bal-prevalence/. Accessed July 23, 2020

16. Witherspoon E. Researchers Build Capacity for Study of Dementia and Risk Factors in Kazakhstan. National Institute of Environmental Health Sciences. Available from: https://www.niehs.nih.gov/research/ programs/geh/geh_newsletter/2019/8/articles/researchers_build_capa city_for_study_of_dementia_and_risk_factors_in_kazakhstan.cfm. Accessed July 22, 2020

17. Prince M, Wimo A, Guerchet M, Ali G, Wu Y, Prina M. The global impact of dementia. World Alzheimer Report. 2015:1-82.

18. Monroe TB, Misra SK, Habermann RC, Dietrich MS, Cowan RL, Simmons SF. Pain reports and pain medication treatment in nursing home residents with and without dementia. Geriatr Gerontol Int. 2014;14(3):541-548. doi:10.1111/ggi.12130

19. Hadjistavropoulos T, Herr K, Prkachin KM, et al. Pain assessment in elderly adults with dementia. Lancet Neurol. 2014;13 (12):1216-1227. doi:10.1016/S1474-4422(14)70103-6

20. Pautex S, Michon A, Guedira M, et al. Pain in severe dementia: self-assessment or observational scales? J Am Geriatr Soc. 2006;54 (7):1040-1045. doi:10.1111/j.1532-5415.2006.00766.x

21. McAuliffe L, Nay R, O’Donnell M, Fetherstonhaugh D. Pain assessment in older people with dementia: literature review. $J$ Adv Nurs. 2009;65(1):2-10. doi:10.1111/j.1365-2648.2008.04861.x 
22. Barry HE, Parsons C, Passmore AP, Hughes CM. Pain in care home residents with dementia: an exploration of frequency, prescribing and relatives' perspectives. Int J Geriatr Psychiatry. 2015;30(1):55-63. doi:10.1002/gps.4111

23. Perceptions of dementia.Dementia Statistics Hub, Alzheimer's Research UK. Available from: https://www.dementiastatistics.org/statis tics/perceptions-of-dementia/. Accessed July 23, 2020
24. Tullo ES, Lee RP, Robinson L, Allan L. Why is dementia different? Medical students' views about deceiving people with dementia. Aging Ment Health. 2015;19(8):731-738. doi:10.1080/13607863.2014.967173

\section{Publish your work in this journal}

The Journal of Pain Research is an international, peer reviewed, open access, online journal that welcomes laboratory and clinical findings in the fields of pain research and the prevention and management of pain. Original research, reviews, symposium reports, hypothesis formation and commentaries are all considered for publication. The manuscript management system is completely online and includes a very quick and fair peer-review system, which is all easy to use. Visit http:// www.dovepress.com/testimonials.php to read real quotes from published authors. 